\title{
Internet-delivered mindfulness for people with depression and chronic pain following spinal cord injury: a randomized, controlled feasibility trial
}

\author{
Jasmine Heath Hearn $\mathbb{D}^{1} \cdot$ Katherine Anne Finlay ${ }^{2}$
}

Received: 17 October 2017 / Revised: 23 February 2018 / Accepted: 27 February 2018 / Published online: 26 March 2018

(c) International Spinal Cord Society 2018

\begin{abstract}
Study design Between-subjects, randomized controlled feasibility study.

Objectives Populations with reduced sensory and motor function are at increased risk of depression, anxiety and pain, and may be less geographically mobile. This study explored the efficacy and feasibility of web-based mindfulness training for people with spinal cord injury (SCI).

Setting UK community sample.

Methods Participants were randomly allocated to an 8-week online mindfulness intervention $(N=36)$, or to internetdelivered psychoeducation $(N=31)$. Depression symptom severity was the primary outcome. Secondary outcomes included anxiety, quality of life (QoL), pain perception, pain catastrophizing and mindfulness. Measures were taken before (T1), at completion of, (T2), and 3 months following the intervention (T3).

Results At T2, ten participants discontinued mindfulness training, and five discontinued psychoeducation. Dropouts were of significantly older age. Nine participants were lost to follow-up. Mindfulness reduced depression significantly more than psychoeducation at T2 (mean difference $=-1.50,95 \% \mathrm{CI}[-2.43,-0.58]$ ) and T3 (mean difference $=-2.34,95 \% \mathrm{CI}$ $[-3.62,-1.10])$. Anxiety, pain unpleasantness and catastrophizing were significantly reduced compared with psychoeducation. Total mindfulness scores, and all facets of mindfulness except observing were significantly higher following mindfulness training. At follow-up, reductions in anxiety and catastrophizing persisted.

Conclusions Internet-delivered mindfulness training offers unique benefits and is viable for people with reduced sensory awareness. Future work should explore the feasibility of combined education and mindfulness training. The use of brief interventions shows promise in maximizing participant retention.
\end{abstract}

\section{Introduction}

Depression is commonly experienced following spinal cord injury (SCI), with a recent meta-analysis indicating a mean prevalence rate of $22.2 \%$ [1], and is associated with chronic

Electronic supplementary material The online version of this article (https://doi.org/10.1038/s41393-018-0090-2) contains supplementary material, which is available to authorized users.

Jasmine Heath Hearn

jasmine.hearn@buckingham.ac.uk

1 The University of Buckingham Medical School, Hunter Street, Buckingham MK18 1EG, UK

2 The Department of Psychology, The University of Buckingham, Hunter Street, Buckingham MK18 1EG, UK pain, with each one often amplifying the other [2]. However, conflict is evident in the literature in terms of interventions to improve such outcomes, with some research trials, based on cognitive behavioural principles, demonstrating improvements in depression [2], yet others reporting no change [3]. Indeed, systematic reviews indicate a need for further evidence of the efficacy of psychological interventions for people with SCI [4]. Similarly, qualitative work indicates that people with SCI desire improved access to psychological interventions [5], but have found the access to and SCI-appropriateness of such interventions difficult to establish.

More recently, focus is being placed upon acceptance and mindfulness-based interventions (MBIs), with the aim to develop present-moment awareness and acceptance, rather than changing thoughts and behaviours [6]. Presentmoment awareness is cultivated through attending to 
internal experiences such as bodily sensations, thoughts and emotions in each moment, in a non-judgmental manner [6]. Approach-focused strategies such as mindfulness are likely to be of value to those with SCI and depression. Though benefits have been documented for people with multiple sclerosis, indicating improvements in quality of life (QoL), mental health and fatigue [7], the utility of MBIs have not been assessed in terms of their appropriateness for those with SCI.

Physical and psychological improvements, such as in anxiety and disability, arising from MBI participation have been documented in various conditions, including chronic back pain [8]. MBIs demonstrate small-tomedium effect sizes on psychological outcomes [9]. Work has shown improvements in depression [10], with preliminary results indicating that mindfulness is associated with reduced experiential avoidance and improved mood in people with SCI [11]. Proposed mechanisms underlying the efficacy of MBIs include cognitive defusion (reduced identification with the contents of one's thoughts), as well as improved self-regulation, emotional, cognitive and behavioural flexibility, and exposure [12]. Heightened awareness of automatic responses to emotions, thoughts and physical states is thought to offer more choice in countering habitual avoidance or denial of difficult emotional or physical states and therefore increase exposure to such states (such as pain). For people with chronic pain, this exposure, combined with the absence of catastrophic consequences, leads to desensitization to pain and reduced negative emotional reactivity [9]. Given that avoidance of negative states is predictive of depression following SCI [11], and that mindfulness training can help to reduce such avoidance, evaluation of the utility of MBIs for improving such important and potentially debilitating outcomes after SCI is required.

Psychoeducation is often included as part of the rehabilitation process following SCI, with NICE guidance recommending timely information on expected outcomes of treatment, return to usual activities and likelihood of permanent effects on QoL, such as pain and psychological outcomes [13]. Psychoeducation has previously been compared to mindfulness training for people with a variety of chronic pain conditions, with subjective wellbeing improving more following mindfulness training, and no differences between groups in improvements for pain interference, pain acceptance and catastrophizing [14]. Despite its promise, no previous work has examined the efficacy of mindfulness training for people with SCI, nor compared mindfulness with psychoeducation as an active control. There therefore exists a need for research to evidence the utility of MBIs in comparison to interventions such as psychoeducation that are offered as part of standard care during and after rehabilitation following SCI.

This study, therefore, aimed to:

- $\quad$ explore the feasibility of eight-week online mindfulness and psychoeducation interventions, specifically retention rates due to the high time commitment required of participants;

- examine the utility of regular engagement with an online mindfulness training intervention as a potential tool for people with SCI to enhance psychological wellbeing.

Hypotheses:

- Mindfulness training will produce greater beneficial changes in psychological wellbeing, and quality of life of people with SCI, compared with psychoeducation.

- As the aim of mindfulness is not to reduce pain, it was anticipated that there would be no differences in painrelated outcomes between mindfulness training and psychoeducation.

\section{Methods}

\section{Design}

This was a between-subjects, single-centre RCT, with depression symptom severity as the primary outcome measure, and secondary outcome measures of anxiety, QoL, pain catastrophizing, mindfulness and pain-related outcomes. A $2 \times 3$ design was employed, addressing the impact of the intervention (two levels: mindfulness training or psychoeducational control group) on each outcome measure over time (three levels: baseline, T1; post-intervention, T2; and 3-month follow-up, T3).

\section{Participants}

Eligible participants were recruited from The National Spinal Injuries Centre, Stoke Mandeville Hospital, UK, and had reduced sensory and motor function arising from SCI for a period of at least 1 year. Participants were over 18 years of age (no upper age limit), had either paraplegia or tetraplegia,had chronic pain for a minimum of 3 months (screened using the LANSS Pain Scale; with a minimum cut-score of 12 [15]), sufficient understanding of English, and internet access for the duration of the study. Exclusion criteria included: presence of any significant cognitive impairment, mental illness or head injury (to reduce the risk of bias or influence on pain perception); presence of any comorbid long-term health conditions that may affect the 
Table 1 Details on mindfulness course content

\begin{tabular}{|c|c|}
\hline Week & Content \\
\hline 1 & $\begin{array}{l}\text { The course began with an introductory video showing participants how to navigate the online server. The first week of the course } \\
\text { started with three variants of the body scan, during which participants draw their attention to various areas of the body, moving } \\
\text { awareness systematically through each area of the body, noticing sensations of the body in a precise and detailed manner, as opposed } \\
\text { to attending to thoughts, ideas or fears about these sensations. }\end{array}$ \\
\hline 2 & $\begin{array}{l}\text { Participants were introduced to breath awareness meditations, alongside a fourth variant of the body scan. Breath awareness } \\
\text { meditations began with a broad awareness of the bodily experience of breathing, becoming increasingly focused on more subtle } \\
\text { aspects of breathing and encouraged participants to notice when their attention wandered away from the meditation. }\end{array}$ \\
\hline 3 & $\begin{array}{l}\text { Mindful movement was introduced, accompanied by body scans. The mindful movement meditation requires that the participant } \\
\text { engage in bodily movements in time with their in- and out-breaths, allowing the pace to be dictated by the natural breath. Altered } \\
\text { movements were designed specific to the abilities of those with SCI, and participants were able to choose which movements to } \\
\text { engage in dependent on their ability. Videos of movements were provided. This week encouraged participants to bring awareness to } \\
\text { their physical activity. This also aimed to teach individuals to pace themselves as they go about daily activities, as opposed to } \\
\text { completing as many as possible while they feel well. }\end{array}$ \\
\hline 4 & $\begin{array}{l}\text { Meditations to foster acceptance and self-compassion were introduced, with participants encouraged to treat themselves with the } \\
\text { kindness that they would treat others with and relax into pain, rather than being distressed by it. }\end{array}$ \\
\hline 5 & $\begin{array}{l}\text { Participants were encouraged to seek out the pleasant things in life, which pain and suffering may have prevented them from } \\
\text { appreciating, by exploring each of their senses. This aimed to allow individuals to become more receptive to positives in their life, no } \\
\text { matter how small. Participants were also encouraged to stop once an hour during daily life to find something positive. Meditations } \\
\text { focused on developing the capacity to notice pleasant aspects of experience. }\end{array}$ \\
\hline 6 & $\begin{array}{l}\text { Encouraging the cultivation of broad, stable, kind and confident awareness continued. Resistance of unpleasant experiences and } \\
\text { grasping on to positive experiences was discouraged, while enjoyment of the depth and breadth of experience, both positive and } \\
\text { negative, was encouraged. In this, participants were asked to acknowledge experiences, and to respond, rather than react, in order to } \\
\text { improve their ability to choose adaptive responses. }\end{array}$ \\
\hline 7 & $\begin{array}{l}\text { This week introduced meditations that encouraged a kind attitude of connectedness and shared experience to oneself, friends and } \\
\text { others (for example, a person with whom the individual holds a difficult relationship with). }\end{array}$ \\
\hline 8 & $\begin{array}{l}\text { During the final week, participants were reminded of all they had learnt during the course. Self-compassion and kindness to others } \\
\text { meditations were practiced for } 3 \text { days, followed by body scan and breath awareness meditations, which were practiced for the } \\
\text { remaining } 3 \text { days. Participants were then presented with a downloadable certificate confirming their completion of } 20 \mathrm{~h} \text { ( } 960 \mathrm{~min} \text { ) of } \\
\text { focused training. }\end{array}$ \\
\hline
\end{tabular}

experience of SCI, or the cause of chronic pain (such as cancer); and previous formal and informal experience of mindfulness practice.

\section{Procedure}

Individuals meeting the inclusion criteria were identified by members of the direct care team at The National Spinal Injuries Centre, and an advertisement was published in various local media outlets, aimed towards people with SCI. Generic letters of invitation (i.e. neutral to the two groups) were sent to all individuals who expressed interest in the study. If they wished to enrol in the study, participants were screened for eligibility and recruited onto the study by members of the direct care team, at which point informed consent was obtained and baseline data collected (T1). Following consent and baseline measure completion, participants were then randomized using an independent, computerized random block randomization programme, to receive either mindfulness training or the psychoeducational intervention. Participants were blinded to their grouping and were not aware of the alternative intervention approach until the study concluded. Participants were provided with the participant information sheet specific to their grouping and given the opportunity to ask questions before the intervention commenced. Participants then undertook their allocated intervention (described in further detail below) for a period of 8 weeks, after which outcome measures were taken (assessors were not blinded to group allocation). Participants in the mindfulness training group did not receive any psychoeducation and vice versa; interventions were delivered in addition to standard care. After the final questionnaires were completed at 3-month follow-up, all participants were debriefed. Upon completion of the study, those in the control group were offered the opportunity to take part in the mindfulness course, and those in the mindfulness group were provided with the psychoeducational materials.

\section{Interventions}

Psychological interventions often necessitate multiple sessions/visits, which may pose a barrier to engagement for people with SCI, given the reduced motor function resulting from injury. However, both MBIs and psychoeducation can be delivered in an online format. In 
collaboration with the Mindfulness Center in Sweden, Breathworks offers an established web-based, 8-week Mindfulness for Health course [16], specifically designed for people with chronic pain and/or illness (also known as Mindfulness-Based Pain Management). The decision to use a web-based course was influenced by the fundamental need to use patient-centred approaches in physical medicine and rehabilitation [17], accounting for factors like geography, transport and motor function. Patients with sustained neurologic conditions, such as SCI, represent populations potentially at the greatest risk of disadvantage due to concomitant physical, functional and support-related limitations which may reduce engagement with health-care services [18]. Thus, to maximize engagement and reduce participant burden, the online course was adopted for this study. Similarly, evidence supports its efficacy for depression [19] and chronic pain intensity and interference [20], making it an appropriate course for the target population.

Participants were instructed to complete the course individually, at times and locations appropriate to their lifestyles. The course delivered two, 10-min audio-guided meditations each day (recorded by trained and accredited mindfulness teachers), on 6 out of 7 days a week, for 8 weeks, totalling $960 \mathrm{~min}$ of practice. Participants were led through a progressive experiential exploration of mindfulness, including: breath awareness, body scanning, kindness and activities for embedding mindfulness in daily life [19] (see Table 1 for more detail on these aspects of the course). One specific aspect of the course that was adapted by the course providers and authors was in mindful movement, designed to promote awareness of physical activity. Mindful movement videos were created to guide participants through a range of small movements that were considered more feasible for people with reduced physical function, including head tilts and wrist rotations. Participants were advised to do mindful movements that were appropriate to their level of function, thus allowing bodily movement within the limits of physical capability. Engagement with the course was monitored by the web host (Mindfulness Center in Sweden), and the authors were notified when participants had completed the course. Participants were provided with a certificate of completion and continue to have unlimited access to the resources online.

Participants in the psychoeducation group received an email once per week for 8 weeks, which provided educational content on SCI and chronic pain in lay terminology and were advised to read these at times and locations suitable for them. This was based on the established elements found in pain management psychoeducation programmes and detailed the epidemiology of SCI and SCI-specific pain, including the biopsychosocial model, the relationship between mood and pain, and the role of stress and unhelpful thoughts. Further topics included options for pain and psychological management (pharmacological and non-pharmacological), and sources of further specific support.

\section{Measures}

Measures were administered via an encrypted online survey before (T1) and after the programme ( 8 weeks; T2) and at 3month follow-up (T3) for both groups. Measures were selected in accordance with recommendations by the Initiative on Methods, Measurement, and Pain Assessment in Clinical Trials (IMMPACT) Group [21]; focus was placed on pain, emotional function, physical function, mindfulness and assessment of compliance with the interventions. All measures selected demonstrate sensitivity to change.

\section{Demographics}

The demographic questionnaire contained nine items pertaining to age, gender, employment and marital status, ethnicity, ASIA impairment score, cause, level of (cervical, thoracic, lumbar, or sacral), and time since, injury.

\section{The Hospital Anxiety and Depression Scale (HADS)}

This is a 14-item Likert scale measure; seven items assess severity of depression and seven items assess severity of anxiety, and responses range from zero to three [22]. Higher scores (range zero to 21 on each outcome) indicate greater symptom severity. It is a reliable measure of severity of depression and anxiety in people without physical restrictions, and those with SCI, without influence of injuryrelated bias (Cronbach's alpha 0.85 for HADS-A, 0.79 for HADS-D) [23]. In the present study HADS-A $\alpha=0.85$, HADS-D $\alpha=0.92$.

\section{Quality of Life (WHOQoL-BREF)}

This 26-item questionnaire measures QoL in four domains, graded on a five-point Likert scale: physical health, psychological health, social relationships and environment [24]. Summed scores range from 0 to 100; higher scores denote greater perceived QoL. Cronbach's alpha for the WHOQoL-BREF for all time points in the present study was between 0.86 and 0.96 , consistent with previous work with people with SCI [25]. 


\section{Five Facet Mindfulness Questionnaire (FFMQ)}

The FFMQ consists of 39 items scored on five-point Likert scales ranging from 1 (never/rarely true) to 5 (very often/always true) [26]. It measures five factors representing mindfulness: observing, describing, acting with awareness, non-judging of inner experience and nonreactivity to inner experience, thus identifying which skills are important predictors of symptom reduction. Facet scores range from 8 to 40, apart from the facet of non-reactivity, which has a range from 7 to 35 . The total maximum score on the FFMQ is therefore 195, with higher scores indicating greater levels of mindfulness. The FFMQ has strong psychometric characteristics, including good reliability with alpha coefficients ranging from 0.72 to 0.92 for all facets and significant incremental validity in previous work [26], and from 0.89 to 0.92 in the present study.

\section{Pain-related measures}

Numerical rating scale (NRS) measures on scales of zero (none) to ten (as bad as it could be), of pain intensity, and pain unpleasantness, were included. The NRS demonstrated good reliability in the present study (pain intensity $\alpha=0.78$, pain unpleasantness $\alpha=0.92$ ).

\section{The Pain Catastrophizing Scale}

This is a 13-item Likert-type scale that measures three domains of catastrophizing, including rumination, magnification and helplessness [27]. Higher scores indicate increased pain-related catastrophizing, with a minimum score of zero and maximum score of 52. Validity and reliability have been demonstrated with a Cronbach's alpha score 0.95 in previous work [28] and in the present study.

\section{Retention rates}

Retention rate was defined as discontinuation and loss to follow-up at 3 months. As the study assessed the utility of regular engagement in mindfulness practice, compliance was defined as completing all $960 \mathrm{~min}$ of the mindfulness course. The maximum attrition rate at follow-up target of $20 \%$ was based on the mean attrition rate from systematic review evidence of mindfulness interventions for people with multiple sclerosis (range 5-43\%) [29].

\section{Statistical methods}

Data were analysed using SPSS version 22. A sample size calculation was performed for the primary outcome measure, depression symptom severity, using $G^{*}$ Power; for a power of $80 \%$, a conservative effect size of 0.25 (based on previous meta-analyses of psychological interventions for people with SCI [30]), two-tailed, with significance set at $p$ $<0.05$, a sample of 42 was necessary, protecting against Type I error. To account for drop-out, a target of 66 participants was set for the sample.

Data were initially examined for distribution normality and outliers. Means and standard deviations were calculated for demographic data. Multiple univariate analyses of covariance (ACNOVAs) were applied to outcome measures in preference to multivariate analyses, controlling for baseline scores for each outcome measure. Correlations were calculated between all outcome measures at $\mathrm{T} 2$ and T3. Confidence intervals and effect sizes are reported throughout.

\section{Results}

A CONSORT flow diagram provides randomization information (see Fig. 1). Participants were recruited between April 2015 and March 2016, with recruitment ending when the target of 66 participants was met (the trial exceeded its required sample size through the use of multiple recruitment strategies). Of the 94 assessed for eligibility, 67 were randomized across the two interventions. Intention-to-treat principles were followed; Little's test indicated that cases were missing at random $\left(X^{2}(3, N=52)=3.03, p=1.00\right)$, and therefore for participants who provided data at $\mathrm{T} 1$ and $\mathrm{T} 2$, missing data points were imputed using multiple imputation. As a result, 67 participants are included in analyses at $\mathrm{T} 1$, and 52 at $\mathrm{T} 2$ and $\mathrm{T} 3$. Both groups were normally distributed for all outcome variables (Shapiro - Wilk; $p>0.05$ ).

\section{Demographic characteristics}

Overall, there were 67 participants with 36 in the intervention group and 31 in the control group. Of the sample, $31(46 \%)$ were male, and mean age was 44.4 years. The majority of the sample were white $(86 \%)$, with $7 \%$ Bangladeshi and $7 \%$ Asian. The location of SCI was lumbar (7\%), thoracic $(55 \%)$ or cervical $(37 \%)$, with road traffic accident the most common cause of injury (40\%), followed by falls $(24 \%)$, non-traumatic causes $(18 \%)$ and sporting injuries (10\%). Participants were most commonly between 2 and 8 years since the onset of their injury (55\%), with $16 \%$ sustaining their injuries within the past 2 years, and $38 \%$ sustaining their injuries over 8 years ago. Participant characteristics can be found in Table 2. 
Fig. 1 CONSORT flow

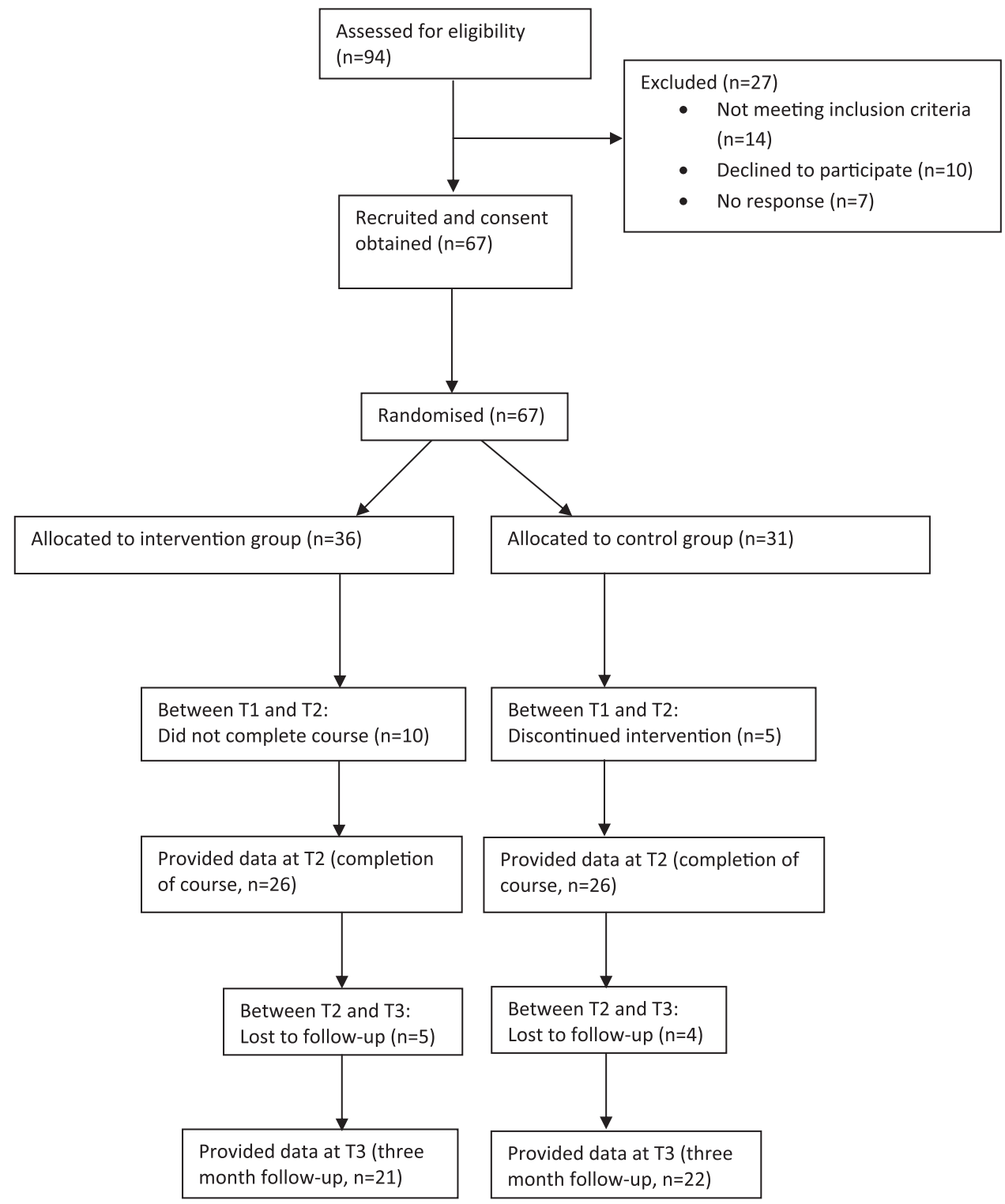

\section{Compliance rate}

The total period taken to screen and enrol the sample size of 67 was 13 months. At T2, a total of ten participants had discontinued the mindfulness training (28\%), indicating a total intervention compliance rate of $72 \%$. Those who dropped out of mindfulness training completed an average of $217 \mathrm{~min}$ of practice (range $40-460 \mathrm{~min}$ ). Five participants discontinued psychoeducation (16\%), indicating a total intervention compliance rate of $84 \%$. Independent samples $t$ tests indicated that those who discontinued were of significantly increased age $(M=49.3, \mathrm{SD}=11.1)$ compared to course completers $(M=43.0, \mathrm{SD}=9.9, p=$ $0.04, d=0.599,95 \%$ CI $[5.22,7.38])$. Further, severity of depression symptoms approached significance, with participants discontinuing the intervention demonstrating increased symptom severity $(M=15.9, \mathrm{SD}=2.4)$ compared to those who completed it $(M=13.8, \mathrm{SD}=4.0, p$ $=0.051, d=0.637,95 \%$ CI $[1.761,2.439])$. There were no other significant differences between those who discontinued and those who completed the interventions on demographics and outcome measures at baseline. Five further participants allocated to mindfulness training (14\%), and four allocated to psychoeducation (13\%), were lost to follow-up at T3, with a total retention rate of $58 \%$ in mindfulness training and $71 \%$ in psychoeducation. There were no differences between study completers and those lost to follow-up on baseline measures or demographic variables. 
Table 2 Demographic characteristics

\begin{tabular}{|c|c|c|c|c|c|c|}
\hline \multirow{4}{*}{ Age } & \multicolumn{2}{|c|}{$\begin{array}{l}\text { Interven- } \\
\text { tion group } \\
(N=36)\end{array}$} & \multicolumn{2}{|c|}{$\begin{array}{l}\text { Comparison } \\
\text { group } \\
(N=31)\end{array}$} & \multicolumn{2}{|c|}{$\begin{array}{l}\text { Total } \\
(N=67)\end{array}$} \\
\hline & $M$ & SD & $M$ & $\mathrm{SD}$ & $M$ & SD \\
\hline & 43.8 & 8.7 & 45.2 & 12.2 & 44.4 & 10.4 \\
\hline & $N$ & $\%$ & $N$ & $\%$ & $N$ & $\%$ \\
\hline \multicolumn{7}{|l|}{ Gender } \\
\hline Male & 17 & 47 & 14 & 45 & 31 & 46 \\
\hline Female & 19 & 53 & 17 & 55 & 36 & 54 \\
\hline \multicolumn{7}{|l|}{ Marital status } \\
\hline Married & 11 & 31 & 9 & 29 & 20 & 30 \\
\hline Widowed & 0 & 0 & 5 & 16 & 5 & 7 \\
\hline Divorced & 3 & 8 & 1 & 3 & 4 & 6 \\
\hline Cohabiting & 3 & 8 & 4 & 13 & 7 & 10 \\
\hline Single & 19 & 53 & 12 & 39 & 31 & 46 \\
\hline \multicolumn{7}{|l|}{ Employment status } \\
\hline Employed, full time & 8 & 22 & 5 & 22 & 13 & 19 \\
\hline Employed, part time & 11 & 28 & 8 & 28 & 19 & 28 \\
\hline Unemployed & 7 & 22 & 8 & 22 & 15 & 22 \\
\hline Retired & 10 & 28 & 10 & 28 & 20 & 30 \\
\hline \multicolumn{7}{|l|}{ Ethnicity } \\
\hline White British & 28 & 78 & 23 & 74 & 51 & 76 \\
\hline White Irish & 3 & 8 & 0 & 0 & 3 & 5 \\
\hline European & 1 & 3 & 1 & 3 & 2 & 3 \\
\hline Other white & 1 & 3 & 0 & 0 & 1 & 2 \\
\hline Bangladeshi & 3 & 8 & 2 & 7 & 5 & 7 \\
\hline Asian & 0 & 0 & 5 & 16 & 5 & 7 \\
\hline \multicolumn{7}{|l|}{ Cause of injury } \\
\hline Road traffic accident & 16 & 44 & 11 & 36 & 27 & 40 \\
\hline Fall & 9 & 25 & 7 & 23 & 16 & 24 \\
\hline Sporting injury & 5 & 14 & 2 & 7 & 7 & 10 \\
\hline Non-traumatic & 6 & 17 & 6 & 19 & 12 & 18 \\
\hline Prefer not to say & 0 & 0 & 5 & 16 & 5 & 8 \\
\hline \multicolumn{7}{|l|}{ Level of injury } \\
\hline $\mathrm{C} 1-\mathrm{C} 8$ & 12 & 33 & 13 & 42 & 25 & 37 \\
\hline $\mathrm{T} 1-\mathrm{T} 5$ & 13 & 36 & 5 & 16 & 18 & 27 \\
\hline $\mathrm{T} 6-\mathrm{T} 12$ & 9 & 25 & 10 & 32 & 19 & 28 \\
\hline $\mathrm{L} 1-\mathrm{L} 5$ & 2 & 6 & 3 & 10 & 5 & 7 \\
\hline \multicolumn{7}{|l|}{ ASIA score } \\
\hline A & 3 & 8 & 6 & 19 & 9 & 13 \\
\hline B & 13 & 36 & 4 & 13 & 17 & 25 \\
\hline $\mathrm{C}$ & 9 & 25 & 10 & 32 & 19 & 28 \\
\hline $\mathrm{D}$ & 11 & 31 & 11 & 36 & 22 & 32 \\
\hline E & 0 & 0 & 0 & 0 & 0 & 0 \\
\hline \multicolumn{7}{|l|}{ Years since injury } \\
\hline $1-2$ & 5 & 14 & 6 & 19 & 11 & 16 \\
\hline $2-4$ & 11 & 31 & 7 & 23 & 18 & 27 \\
\hline $4-8$ & 11 & 31 & 8 & 26 & 19 & 28 \\
\hline $8-12$ & 3 & 8 & 3 & 10 & 6 & 9 \\
\hline $12-15$ & 3 & 8 & 4 & 13 & 7 & 10 \\
\hline $15+$ & 3 & 8 & 3 & 10 & 6 & 19 \\
\hline
\end{tabular}

n.b. percentages have been rounded

\section{Effect of the intervention}

Analysis of covariance (ANCOVA) was conducted for all outcome measures with baseline scores set as covariates in each analysis. Additionally, level of injury and ASIA scores were also controlled for, given that there were more people in the mindfulness training group with levels of injury at $\mathrm{T} 1$ - T5 and ASIA B scores compared with the psychoeducation group. At T2, significant improvements in favour of mindfulness training $(p<0.05)$ were found for severity of depression (partial eta squared $\left(\eta_{\mathrm{p}}^{2}\right)=0.184$; mean between group difference $=-1.50,95 \%$ CI $[-2.43,-0.58])$, anxiety $\left(\eta_{\mathrm{p}}^{2}=0.137\right.$; mean between group difference $=-1.50$, $95 \%$ CI $[-2.60,-0.40])$, pain unpleasantness $\left(\eta_{p}^{2}=0.137\right.$; mean between group difference $=-0.96,95 \%$ CI $[-1.67$, $-0.25])$, and pain catastrophizing $\left(\eta_{\mathrm{p}}^{2}=0.110\right.$; mean between group difference $=-2.26,95 \%$ CI $[-4.14$, $-0.38])$.

Significant differences at T2 were also noted for mindfulness facets of acting with awareness $\left(\eta_{\mathrm{p}}^{2}=0.220\right.$; mean between group difference $=1.60,95 \%$ CI $[0.716,2.49]$ ), describing $\left(\eta_{\mathrm{p}}^{2}=.098\right.$; mean between group difference $=$ $1.43,95 \%$ CI $[0.16,2.69])$, non-judging $\left(\eta_{\mathrm{p}}^{2}=0.081\right.$; mean between group difference $=1.20,95 \%$ CI $[0.01,2.38])$, and non-reactivity to inner experience $\left(\eta_{\mathrm{p}}^{2}=0.167\right.$; mean between group difference $=1.36,95 \% \mathrm{CI}[0.47,2.25])$, and the total FFMQ score $\left(\eta_{\mathrm{p}}^{2}=0.277\right.$; mean between group difference $=6.25,95 \%$ CI $[3.28,9.21])$. There were no significant group differences at T2 for any aspect of QoL, pain intensity, and mindfulness facets of observing and nonjudging. At T3, significant group differences $(p<0.05)$ persisted for severity of depression $\left(\eta_{\mathrm{p}}^{2}=0.223\right.$; mean between group difference $=-2.34,95 \%$ CI [-3.62, $-1.10])$, anxiety $\left(\eta_{\mathrm{p}}^{2}=0.112\right.$; mean between group difference $=-1.31,95 \%$ CI $[-2.39,-0.23])$, and pain catastrophizing $\left(\eta_{\mathrm{p}}^{2}=0.239\right.$; mean between group difference $=$ $-3.77,95 \%$ CI $[-5.75,-1.80])$. Means and standard deviations for each outcome measure at each time point are reported in Table 3. Results of the ANCOVAs are reported in Tables 4 and 5. Spearman's rho correlation matrixes for all outcome variables are provided for $\mathrm{T} 2$ and $\mathrm{T} 3$ as supplementary files (Tables 6 and 7).

\section{Discussion}

This is the first study exploring the effects of an 8-week, internet-delivered mindfulness training intervention for people with reduced sensory and motor function arising from SCI. Compared to psychoeducation, online mindfulness training offered greater improvements in symptoms of depression and anxiety, pain catastrophizing, and specific facets of mindfulness (describing, acting with awareness, non-reactivity to inner experience, and total scores) at completion of the intervention. At follow-up, depression and anxiety severity and pain catastrophizing demonstrated a persistent decrease and were significantly 
Table 3 Self-report outcome measures: means and standard deviations

\begin{tabular}{|c|c|c|c|c|c|c|c|}
\hline & & \multicolumn{3}{|c|}{ Intervention } & \multicolumn{3}{|l|}{ Control } \\
\hline & & $\begin{array}{l}\mathrm{T} 1 \\
(N=36)\end{array}$ & $\begin{array}{l}\mathrm{T} 2 \\
(N=26)\end{array}$ & $\begin{array}{l}\mathrm{T}^{\mathrm{a}} \\
(N=26)\end{array}$ & $\begin{array}{l}\mathrm{T} 1 \\
(N=31)\end{array}$ & $\begin{array}{l}\mathrm{T} 2 \\
(N=26)\end{array}$ & $\begin{array}{l}\mathrm{T}^{\mathrm{a}} \\
(N=26)\end{array}$ \\
\hline \multicolumn{8}{|l|}{ WHOQoL-BREF } \\
\hline \multirow[t]{2}{*}{ Physical } & Mean & 52.3 & 54.6 & 55.0 & 52.9 & 55.8 & 56.8 \\
\hline & SD & 5.0 & 5.0 & 6.2 & 6.4 & 5.2 & 5.2 \\
\hline \multirow[t]{2}{*}{ Psychological } & Mean & 56.8 & 61.2 & 61.2 & 58.5 & 61.9 & 60.6 \\
\hline & SD & 6.7 & 5.5 & 5.8 & 6.9 & 7.3 & 6.5 \\
\hline \multirow[t]{2}{*}{ Social } & Mean & 58.6 & 65.4 & 69.1 & 57.2 & 63.0 & 65.2 \\
\hline & SD & 8.1 & 7.9 & 10.8 & 8.6 & 7.3 & 9.4 \\
\hline \multirow[t]{2}{*}{ Environmental } & Mean & 63.2 & 64.4 & 65.3 & 56.7 & 60.2 & 62.4 \\
\hline & SD & 7.3 & 6.6 & 7.9 & 8.2 & 8.0 & 8.0 \\
\hline \multicolumn{8}{|l|}{ HADS } \\
\hline \multirow[t]{2}{*}{ Depression } & Mean & 15.6 & 12.6 & 11.3 & 12.7 & 11.8 & 11.3 \\
\hline & SD & 2.9 & 3.2 & 3.6 & 4.1 & 3.2 & 3.5 \\
\hline \multirow[t]{2}{*}{ Anxiety } & Mean & 14.5 & 11.6 & 11.2 & 13.1 & 12.0 & 11.6 \\
\hline & SD & 3.9 & 3.2 & 3.2 & 4.1 & 3.7 & 3.7 \\
\hline \multirow[t]{2}{*}{ Pain intensity } & Mean & 6.5 & 5.0 & 4.7 & 7.3 & 5.6 & 5.5 \\
\hline & SD & 2.1 & 1.4 & 1.6 & 2.0 & 2.2 & 2.3 \\
\hline \multirow[t]{2}{*}{ Pain unpleasantness } & Mean & 7.0 & 5.0 & 5.0 & 7.9 & 6.4 & 6.1 \\
\hline & SD & 1.8 & 1.2 & 1.5 & 2.1 & 2.0 & 2.3 \\
\hline \multirow[t]{2}{*}{ PCS } & Mean & 29.0 & 26.1 & 24.9 & 36.5 & 34.5 & 34.6 \\
\hline & SD & 6.2 & 6.2 & 6.1 & 9.0 & 9.5 & 9.6 \\
\hline \multirow[t]{2}{*}{ Mindfulness total (FFMQ) } & Mean & 110.7 & 121.6 & 121.6 & 120.2 & 122.2 & 123.3 \\
\hline & SD & 27.5 & 20.7 & 20.3 & 31.7 & 31.7 & 32.3 \\
\hline \multirow[t]{2}{*}{ Observing } & Mean & 20.3 & 22.2 & 22.8 & 21.9 & 23.0 & 23.7 \\
\hline & SD & 6.9 & 5.7 & 6.3 & 6.9 & 6.5 & 7.0 \\
\hline \multirow[t]{2}{*}{ Describing } & Mean & 19.8 & 21.3 & 21.2 & 23.4 & 23.4 & 23.6 \\
\hline & SD & 6.4 & 5.7 & 7.2 & 7.2 & 7.7 & 8.2 \\
\hline \multirow[t]{2}{*}{ Acting with awareness } & Mean & 23.0 & 25.3 & 25.1 & 24.6 & 24.6 & 25.1 \\
\hline & SD & 7.0 & 5.4 & 6.0 & 6.8 & 7.0 & 7.8 \\
\hline \multirow[t]{2}{*}{ Non-judging } & Mean & 23.3 & 25.6 & 25.9 & 24.9 & 25.2 & 25.5 \\
\hline & SD & 6.0 & 4.8 & 6.8 & 6.7 & 6.8 & 8.4 \\
\hline \multirow[t]{2}{*}{ Non-reactivity } & Mean & 24.3 & 27.1 & 26.6 & 25.3 & 26.0 & 25.4 \\
\hline & SD & 6.4 & 4.8 & 5.1 & 6.8 & 6.6 & 6.2 \\
\hline
\end{tabular}

WHOQoL-BREF World Health Organization Quality of Life Brief Scale, HADS Hospital Anxiety and Depression Scale, PCS Pain Catastrophizing Scale, FFMQ Five Facet Mindfulness Questionnaire

${ }^{a}$ N.B. pooled means and standard deviations

lower in the mindfulness training group compared to the control group. Pain unpleasantness, severity of anxiety, the WHOQoL subscales of physical and psychological QoL, and the FFMQ facet of non-reactivity to inner experience significantly predicted depression severity at intervention completion, while at follow-up, anxiety and pain unpleasantness significantly predicted depressive symptom severity. At follow-up the largest effect size was demonstrated for improvements in symptoms of depression, indicating a strong relationship between engagement in mindfulness training, and improvement in this outcome.

The intervention completion rate was high (average $78 \%$ ), indicating that the interventions were viable and could be successfully embedded into daily life following SCI. However, the drop-out rate was higher in mindfulness training (28\%) compared with psychoeducation (16\%). This may be reflective of the difference in commitment required by the interventions, with mindfulness training requiring twice daily participation in mindfulness practices, and 
Table 4 Analysis of covariance for group effects at $\mathrm{T} 2(N=52)$

\begin{tabular}{|c|c|c|c|c|c|}
\hline Measure & $F$ & $p$ value & $\eta_{\mathrm{p}}^{2}$ & $\begin{array}{l}\text { Mean difference T2 } \\
\text { (mindfulness - control) }\end{array}$ & $\begin{array}{l}95 \% \text { CI for T2 mean } \\
\text { difference (lower, upper) }\end{array}$ \\
\hline \multicolumn{6}{|l|}{ HADS } \\
\hline Depression (0-21) & 10.61 & $0.002^{*}$ & 0.184 & -1.50 & $-2.43,-0.58$ \\
\hline Anxiety $(0-21)$ & 7.46 & $0.009^{*}$ & 0.137 & -1.50 & $-2.60,-0.40$ \\
\hline \multicolumn{6}{|l|}{ WHOQoL-BREF } \\
\hline Physical (0-100) & 0.61 & 0.438 & 0.013 & -0.63 & $-2.25,0.99$ \\
\hline Psychological (0-100) & 2.08 & 0.155 & 0.043 & 1.25 & $-0.49,2.99$ \\
\hline Social (0-100) & 1.11 & 0.298 & 0.023 & 1.56 & $-1.42,4.54$ \\
\hline Environment $(0-100)$ & 0.17 & 0.898 & 0.000 & 0.11 & $-1.55,1.77$ \\
\hline Pain intensity $(0-10)$ & 0.60 & 0.442 & 0.013 & -0.39 & $-1.39,0.62$ \\
\hline Pain unpleasantness $(0-10)$ & 7.44 & $0.009^{*}$ & 0.137 & -0.96 & $-1.67,-0.25$ \\
\hline PCS $(0-52)$ & 5.83 & $0.020^{*}$ & 0.110 & -2.26 & $-4.14,-0.38$ \\
\hline \multicolumn{6}{|l|}{ FFMQ } \\
\hline Total (39-195) & 17.97 & $0.000^{*}$ & 0.277 & 6.25 & $3.28,9.21$ \\
\hline Observing (8-40) & 3.83 & 0.056 & 0.075 & 0.76 & $-0.02,1.55$ \\
\hline Describing (8-40) & 5.13 & $0.028 *$ & 0.098 & 1.43 & $0.16,2.69$ \\
\hline Acting with awareness $(8-40)$ & 13.23 & $0.001 *$ & 0.220 & 1.60 & $0.716,2.49$ \\
\hline Non-judging (8-40) & 4.15 & $0.047 *$ & 0.081 & 1.20 & $0.01,2.38$ \\
\hline Non-reactivity (7-35) & 9.41 & $0.004 *$ & 0.167 & 1.36 & $0.47,2.25$ \\
\hline
\end{tabular}

CI confidence interval, WHOQoL-BREF World Health Organization Quality of Life Brief Scale, HADS Hospital Anxiety and Depression Scale, $P C S$ Pain Catastrophizing Scale, FFMQ Five Facet Mindfulness Questionnaire

${ }^{*} p<0.05$

psychoeducation requiring participants to read educational materials once per week. Further, mindfulness training required active participation and intrinsic motivation to $\log$ on for twice daily mindfulness practice, while participation in the psychoeducation group involved more passive participation. The increased time commitment and active engagement required in mindfulness training may have acted as a barrier to engagement [31], while provision of materials via email in the psychoeducation group may have reduced participant burden.

People who discontinued the intervention were likely to display more severe symptoms of depression, and were of increased age, suggesting that adherence to the intervention was more difficult for these subgroups. Depression severity acts as a predictor for drop-out in internetdelivered interventions [32]. Increased time and effort required of those with more severe psychological difficulties, who may have past experience of unsuccessful treatment, may result in difficulties in continuing an intervention [31]. Similarly, increased age may act as a barrier to engagement through potential loss of social support for continuation in an intervention, as well as differential use of the internet [32]. The present study suggests a need to establish more effective methods of supporting people with comorbid conditions, such as depression and SCI, and those who are of older age with physical disabilities, in order to facilitate improved engagement in psychological interventions and improved outcomes.

In the present study, improvements seen in symptoms of depression and anxiety are supportive of work by Skinner et al. [11], who found a negative correlation between mindfulness and depression for people with SCI, a relationship mediated by avoidance. This suggests that cognitive reappraisal initiated through mindfulness training may have increased acceptance and influenced the way in which participants responded to emotions and thoughts associated with depression and anxiety, such as reduced experiential and behavioural avoidance. These results echo the beneficial effects noted in previous trials with people with multiple sclerosis [9] and chronic back pain [8]. They also support previous evaluation of the course [19], which demonstrated immediate improvements following completion in measures of depression, positive outlook, catastrophizing, activities engagement and pain acceptance, with medium-tolarge effect sizes supporting each result. Online mindfulness training may therefore initiate changes in the way that participants appraised emotions, thoughts and events, with beneficial effects for emotional aspects of life after SCI, which is echoed by the results of the present study, particularly in relation to depression and catastrophic thinking. 
Table 5 Analysis of covariance for group effects at T3 $(N=52)$

\begin{tabular}{|c|c|c|c|c|c|}
\hline Measure & $F$ & $p$ value & $\eta_{\mathrm{p}}^{2}$ & $\begin{array}{l}\text { Mean difference T3 } \\
\text { (mindfulness - control) }\end{array}$ & $\begin{array}{l}95 \% \text { CI for } \mathrm{T} 3 \text { mean } \\
\text { difference (lower, upper) }\end{array}$ \\
\hline \multicolumn{6}{|l|}{ HADS } \\
\hline Depression (0-21) & 13.55 & $0.001 *$ & 0.223 & -2.34 & $-3.62,-1.10$ \\
\hline Anxiety (0-21) & 5.99 & $0.023^{*}$ & 0.112 & -1.31 & $-2.39,-0.23$ \\
\hline \multicolumn{6}{|l|}{ WHOQoL-BREF } \\
\hline Physical (0-100) & 1.40 & 0.330 & 0.028 & -1.33 & $-3.93,1.27$ \\
\hline Psychological (0-100) & 3.48 & 0.119 & 0.068 & 2.08 & $-0.18,4.33$ \\
\hline Social $(0-100)$ & 1.78 & 0.224 & 0.036 & 3.14 & $-1.72,8.00$ \\
\hline Environment (0-100) & 0.29 & 0.674 & 0.006 & -0.67 & $-2.36,2.54$ \\
\hline Pain intensity $(0-10)$ & 1.01 & 0.345 & 0.021 & -0.48 & $-1.45,0.57$ \\
\hline Pain unpleasantness $(0-10)$ & 1.52 & 0.239 & 0.031 & -0.54 & $-1.42,0.34$ \\
\hline PCS $(0-52)$ & 14.87 & $.001 *$ & .239 & -3.77 & $-5.75,-1.80$ \\
\hline \multicolumn{6}{|l|}{ FFMQ } \\
\hline Total (39-195) & 3.00 & 0.225 & 0.058 & 4.49 & $-1.64,10.61$ \\
\hline Observing (8-40) & 0.82 & 0.551 & 0.017 & 0.55 & $-1.17,2.27$ \\
\hline Describing (8-40) & 1.15 & .517 & 0.023 & 0.91 & $-1.54,3.35$ \\
\hline Acting with awareness $(8-40)$ & .68 & .551 & 0.014 & 0.72 & $-1.66,3.10$ \\
\hline Non-judging (8-40) & 0.92 & 0.595 & 0.019 & 1.09 & $-2.34,4.53$ \\
\hline Non-reactivity (7-35) & 3.85 & 0.135 & 0.073 & 1.48 & $-0.26,3.23$ \\
\hline
\end{tabular}

CI confidence interval, WHOQoL-BREF World Health Organization Quality of Life Brief Scale, HADS Hospital Anxiety and Depression Scale, $P C S$ Pain Catastrophizing Scale, FFMQ Five Facet Mindfulness Questionnaire

$* p<0.05$

Pain catastrophizing was significantly reduced by mindfulness training, over and above the observed change in the control group, an improvement seen immediately upon completion of the course, and at 3-month follow-up. It is likely that cognitive reappraisal or 'uncoupling' the sensory experience of pain from the emotional and cognitive experience of pain occurred, which decreased negative emotional responses to its presence. Recent work supports this, indicating that the way that people with SCI think and talk about chronic pain may reflect catastrophic thinking, and increase the attention paid to pain [33]. The training programme adopted in the present study has been specifically developed for people with chronic physical health conditions, which may mitigate this contradiction in results. Such improvements could therefore be further enhanced with MBIs that are specifically targeted for populations with reduced sensory awareness and motor function. The reduction in catastrophizing in the present study suggests that mindfulness training initiated cognitive reappraisal, interrupting the amount of focus placed upon pain. This is supported by the change in perception of pain unpleasantness evidenced in the present study, highlighting potentially increased psychological flexibility and ability to hold an awareness of pain without negative judgement, or getting embroiled in pain-related cognitions and attempts to control pain.
Pain unpleasantness was reduced to a greater extent in the mindfulness training group compared to psychoeducation at completion of the course, but not at follow-up. Mindfulness training may instigate cognitive reappraisal of personal experiences and a change in perspective of the self; this may occur through a process of learning about the relationship between mood and pain and thus a change in the perceived meaning of pain [34]. Further, decreases in perceived barriers to emotional and pain management, and increased acceptance of pain and personal experience may also play a role in reducing pain perception. However, future work should aim to describe the mechanisms underlying changes in perceived pain unpleasantness and explore the extent to which reduced perception of pain unpleasantness requires continued engagement with mindfulness practice.

In summary, the results of the present study show promise, with internet-delivered mindfulness improving some outcomes to a higher degree than standard psychoeducation and demonstrating its utility as an intervention for improving mindful awareness and psychological outcomes for people with reduced sensory and motor function. This study, therefore, provides a foundation on which to explore the impact of MBIs for other neurological groups, and provides rationale for the development of MBIs and mindfulness meditations sensitive to the specific needs of people with neurological deficits. 


\section{Limitations and future research}

This study explored the feasibility and impact of an 8-week mindfulness training intervention on depressive symptom severity, anxiety, QoL and pain-related outcomes in people with SCI. The overall study drop-out rate was high (36\%) and the results are representative of people who have engaged with all $960 \mathrm{~min}$ of mindfulness practice. A convenience sample recruited through advertisement of the study in media outlets could pose risk of selection bias, with those expressing interest more likely to demonstrate improvements in targeted outcomes. Those who completed the course and engaged fully with the educational materials may have been more motivated to engage in self-care, and therefore may be more likely to experience positive change. It would be of benefit to follow up those who discontinued mindfulness training, exploring the effects on wellbeing and their motivations for dropping out. This would provide information to enhance adherence, reduce barriers to training, and establish the relationship between mindfulness practice, and health-related outcomes.

The present study marks the first step in investigating the benefit of mindfulness for people with SCI, highlighting immediate benefits. Future work is required to rigorously evaluate the mechanisms of change underlying the effects of specific aspects of mindfulness and psychoeducation on psychosocial outcomes after SCI. Similarly, work should explore the feasibility of combined education and mindfulness training, for optimum benefit, and the use of brief interventions to maximize participant retention.

Acknowledgements This trial is registered with the ISRCTN, reference number ISRCTN14165286. The authors would like to express their thanks to all of the participants for their involvement and feedback throughout the project, Breathworks for their provision of the course, and staff at The National Spinal Injuries Centre for their ongoing support.

\section{Compliance with ethical standards}

Conflict of interest The authors declare that they have no conflict of interest.

Ethical approval This study was approved by The University of Buckingham School of Science and Postgraduate Medicine Ethics Committee, the NHS Health Research Authority (ref: 14/SC/1424), the local Research and Development office, and The National Spinal Injuries Centre. The trial was registered prospectively with an International Standard Randomised Controlled Trial Number (ISRCTN14165286).

Informed consent All participants provided informed consent and were debriefed following completion of the study. Ongoing support was offered by the researchers, and staff from the centre providing the online course. All patient identifiable information and their corresponding data files were stored separately on a password-protected computer at The Psychology Department at the University of Buckingham. All applicable institutional and governmental regulations concerning the ethical use of human volunteers were followed during the course of this research.

\section{References}

1. Williams R, Murray A. Prevalence of depression after spinal cord injury: a meta-analysis. Arch Phys Med Rehabil. 2015;96:133-40.

2. Ullrich PM, Lincoln RK, Tackett MJ, Miskevics S, Smith BM, Weaver FM. Pain, depression, and health care utilization over time after spinal cord injury. Rehabil Psychol. 2013;58:158-65.

3. Norrbrink Budh C, Kowalski J, Lundeberg T. A comprehensive pain management programme comprising educational, cognitive and behavioural interventions for neuropathic pain following spinal cord injury. J Rehabil Med. 2006;38:172-80.

4. Boldt I, Eriks-Hoogland I, Brinkhof MW, de Bie R, Joggi D, von Elm E. Non-pharmacological interventions for chronic pain in people with spinal cord injury. Cochrane Database Syst Rev. 2014. http://onlinelibrary.wiley.com/doi/10.1002/14651858. CD009177.pub2/abstract.

5. Hearn JH, Cotter I, Fine P, Finlay KA. Living with chronic neuropathic pain after spinal cord injury: an interpretative phenomenological analysis of community experience. Disabil Rehabil. 2015;37:2203-11.

6. Kabat-Zinn J. Full catastrophe living: using the wisdom of your body and mind to face stress, pain, and illness. New York, NY: Dell; 1990.

7. Simpson R, Booth J, Lawrence M, Byrne S, Mair F, Mercer S. Mindfulness based interventions in multiple sclerosis-a systematic review. Bmc Neurol. 2014;14:1-9.

8. Kingston J, Chadwick D, Meron C, Skinner TC. A pilot randomized comparison trial investigating the effect of mindfulness practice on pain tolerance, psychological well-being, and physiological activity. J Psychosom Res. 2007;62:297-300.

9. Baer RA. Mindfulness training as a clinical intervention: a conceptual and empirical review. Clin Psychol: Sci Pract. 2003;10:125-43.

10. Ma SH, Teasdale JD. Mindfulness-based cognitive therapy for depression: replication and exploration of differential relapse prevention effects. J Consult Clin Psychol. 2004;72:31-40.

11. Skinner TC, Roberton T, Allison GT, Dunlop S, Bucks RS. Experiential avoidance, mindfulness and depression in spinal cord injuries: a preliminary study. Aust $\mathrm{J}$ Rehabil Couns, The. 2010;16:27-35.

12. Shapiro SL, Carlson LE, Astin JA, Freedman B. Mechanisms of mindfulness. J Clin Psychol. 2006;62:373-86.

13. National Institute for Health and Care Excellence. Spinal injury: assessment and initial management. NICE Guideline [NG41]. NICE, London. 2016. https://www.nice.org.uk/guidance/ng41/cha pter/Recommendations\#information-and-support-484for-patientsfamily-members-and-carers. Accessed 23 Jan 2018.

14. Dowd H, Hogan MJ, McGuire BE, Davis MC, Sarma KM, Fish $\mathrm{RA}$, et al. Comparison of online mindfulness-based cognitive therapy intervention with online pain management psychoeducation; a randomized controlled study. Clin J Pain. 2015;31:517-27.

15. Bennett M. The LANSS Pain Scale: the Leeds assessment of neuropathic symptoms and sign. Pain. 2001;92:147-57.

16. Burch V, Penman D. Mindfulness for health: a practical guide to relieving pain, reducing stress and restoring wellbeing. London: Piatkus; 2013.

17. Donnelly C, Eng JJ, Hall J, Alford L, Giachino R, Norton K, et al. Client-centred assessment and the identification of meaningful treatment goals for individuals with a spinal cord injury. Spinal Cord. 2008;42:302-7. 
18. Tenforde AD, Hefner JE, Kodish-Wachs JE, Iaccarino MA, Paganoni S. Telehealth in physical medicine and rehabilitation: a narrative review. Phys Med Rehabil. 2017;9:S51-S58.

19. Cusens B, Duggan GB, Thorne K, Burch V. Evaluation of the Breathworks mindfulness-based pain management programme: effects on well-being and multiple measures of mindfulness. Clin Psychol Psychother. 2010;17:63-78.

20. Henriksson J, Wasara E, Ronnlund M. Effects of eight-week webbased mindfulness training on pain intensity, pain acceptance, and life satisfaction in individuals with chronic pain. Psychol Rep. 2016;119:586-607.

21. Turk DC, Dworkin RH, Allen RR, Bellamy N, Brandenburg N, Carr DB, et al. Core outcome domains for chronic pain clinical trials: IMMPACT recommendations. Pain. 2003;106:337-45.

22. Zigmond AS, Snaith RP. The hospital anxiety and depression scale. Acta Psychiatr Scand. 1983;67:361-70.

23. Woolrich RA, Kennedy P, Tasiemski T. A preliminary psychometric evaluation of the Hospital Anxiety and Depression Scale (HADS) in 963 people living with a spinal cord injury. Psychol Health Med. 2006;11:80-90.

24. World Health Organization. Development of the World Health Organization WHOQOL-BREF quality of life assessment. The WHOQOL Group. Psychol Med. 1998;28:551-8.

25. Lude P, Kennedy P, Elfström M, Ballert CS. Quality of life in and after spinal cord injury rehabilitation: a longitudinal multicenter study. Top Spinal Cord Inj Rehabil. 2014;20:197-207.

26. Baer RA, Smith GT, Hopkins J, Krietemeyer J, Toney L. Using self-report assessment methods to explore facets of mindfulness. Assessment. 2006;13:27-45.
27. Sullivan MJL, Bishop SR, Pivik J. The Pain Catastrophizing Scale: development and validation. Psychol Assess. 1995;7:524-32.

28. Osman A, Barrios FX, Kopper BA, Hauptmann W, Jones J, O'Neill E. Factor structure, reliability, and validity of the Pain Catastrophizing Scale. J Behav Med. 1997;20:589-605.

29. Simpson R, Booth J, Lawrence M, Byrne S, Mair F, Mercer S. Mindfulness based interventions in multiple sclerosis-a systematic review. Bmc Neurol. 2014;14:15.

30. Dorstyn D, Mathias J, Denson L. Efficacy of cognitive behaviour therapy for the management of psychological outcomes following spinal cord injury: a meta-analysis. J Health Psychol. 2011;16:374-91.

31. Melville KM, Casey LM, Kavanagh DJ. Dropout from internetbased treatment for psychological disorders. Br J Clin Psychol. 2010;49:455-71.

32. Christensen H, Griffiths KM, Farrer L. Adherence in internet interventions for anxiety and depression: systematic review. J Med Internet Res. 2009;11:e13

33. Hearn JH, Finlay KA, Fine PA. The devil in the corner: a mixed-methods study of metaphor use by those with spinal cord injury-specific neuropathic pain. $\mathrm{Br} \mathrm{J}$ Health Psychol. 2016;21:973-88.

34. Hölzel BK, Lazar SW, Gard T, Schuman-Olivier Z, Vago DR, Ott U. How does mindfulness meditation work? Proposing mechanisms of action from a conceptual and neural perspective. Perspect Psychol Sci. 2011;6:537-59. 